Classification of the Causes of EnlargeMENT OF THE LYMPH GLANDS

I. Infective states :

(a) Various known organisms such as T.B., typhoid, typhus, syphilis, scarlatina, diphtheria, chancroid, gonorrhoea, staphylococcal and streptococcal infections, erysipelas, malaria, anthrax, glanders and tripanosomiasis.

(b) Virus and query virus infections, such as rubella, varicella, variola, infective mononucleosis, Hodgkin's 'disease, lymphogranuloma venereum and Boeck's sarcoid.

(c) Fungus infections such as trichophyton, actinomycosis, torula histolitica, sporotrichosis and coccidiodal granuloma.
2. Lymphatism in children, perhaps from debility and upper respiratory tract infection.

3. Skin conditions, including the various dermatoses arising from chemical agencies, infection, or both.

4. Neoplasms :

(a) Primary :

Lymphatic leukaemia.

Hodgkin's disease.

Lymphosarcoma.

(b) Secondary from tumours arising in various parts of the body.

\section{BIBLIOGRAPHY}

HARRISON, L. W., "The Diagnosis and Treatment of Venereal Diseane in General Practice," London.

\title{
THE DIAGNOSIS, PROGNOSIS AND SYMPTOMATIC TREATMENT OF CARCINOMA OF THE STOMACH
}

\author{
By N. S. Plummer, M.D., F.R.C.P. \\ Physician with Charge of Out Patients, Charing Cross Hospital
}

Carcinoma of the stomach is usually so silent in its onset, its radical cure is consequently so disappointing in effect, that many clinicians despair of ever curing a patient suffering from this disease. It is probably true to say that in the majority of cases when the diagnosis is made on an appreciable filling defect on roentgenological examination, as it usually is, the patient is already past hope of radical cure. Our duty, however, is clear; we must employ all available means to discover the early case for the surgeon, and, in the filling defect stage mentioned above, do all we can to prolong a potentially useful life by well judged surgery and to alleviate suffering by medical means. The importance of the problem is shown by the fact that a third of all carcinomas in men involve the stomach and, in women, stomach carcinomata constitute a fifth of the total cases.

Diagnosis resolves itself into two parts. When is carcinoma of the stomach to be suspected, and if suspected, how is it to be confirmed ?
With an age distribution of 4 per cent. under 30 years, of 7 per cent. between 30 and 40 years and the rest over 40 years, it is clear that we must concentrate on the age group over 40 , especially as the younger the patient the more rapid and malignant is the type of growth, so making the outlook even more hopeless.

Of local symptoms what type of dyspepsia does carcinoma of the stomach produce? The answer is any type of dyspepsia, ranging from mild epigastric discomfort with eructation of wind immediately after food to continuous severe pain or regular intermittent pain at an interval after food as in duodenal ulcer. The main feature of carcinoma dyspepsia is that in about 80 per cent. of cases it begins after the age of 40 in a person not previously subject to indigestion. Thus gastric symptoms in a patient over 40 years of age which last for more than seven to ten days should be fully investigated immediately. In a minority of cases in which there is a preceding dyspepsia for a year or more, due possibly to ulcer or gastritis, the 
symptoms may change in one of several ways ; a regular intermittent dyspepsia may become continuous, the pain may become more severe, vomiting may be more noticeable and, of course, anorexia, loss of weight and anaemia are most suggestive. All such suggestive features call for radiological investigation and other means of confirmation of the suspicion of new growth, despite the fact that, in most instances, there has merely been an exacerbation or simple complication of the primary condition.

The site of the growth is an important factor. A growth of the body of the stomach may reach a large size before producing symptoms, while of the pyloric end there may be symptoms of pyloric obstruction relatively early. A carcinoma at the fundus may give dysphagia if the oesophageal opening is involved, but this is usually a late symptom. I well remember an example of this where the radiological appearance and even the sensation with the passage of a mercury tube were typical of achalasia of the cardia, until the finding of an enlarged gland behind the lower end of the sternomastoid muscle revealed the true diagnosis.

Almost any symptom referred to the abdomen may have its origin in carcinoma of the stomach ; for example an otherwise unexplained diarrhoea or constipation coming on in a person over the age of 40 may indicate that the carcinoma is involving the pylorus, either fixing it in an open position resulting in rapid emptying and consequent diarrhoea, or, by narrowing the outlet, producing constipation, especially if the food intake is reduced. Secondary deposits may give jaundice, ascites, bowel or urinary or other symptoms.

More remotely a carcinoma of the stomach may be heralded by hypochromic or macrocytic anaemia, by enlarged glands in the neck or axilla, by thrombophlebitis in the leg, or peripheral neuritis, or by numerous other clinical manifestations.

It must be admitted that nearly all the symptoms so far mentioned are late in terms of operability. Occasionally an early carcinoma of the stomach is found on radiological examination or at gastroscopy, done for some other reason, when the patient will admit to no gastric symptoms at all, as in the following case. Plummer (I93I).

A man of 63 years had been treated for Addison's anaemia for six years when he was readmitted for enlarged glands in the root of the neck on the right side. A filling defect in the pyloric vestibule on the radiogram seemed to confirm the diagnosis of neoplasm of the stomach despite the absence of any gastric complaint. Biopsy of the gland, however, showed it to be tuberculous. At operation there was a carcinomatous ulcer half encircling the pyloric region and about 2 ins. long. Partial gastrectomy was performed. Later the man became maniacal, the wound burst open and he died in coma a few days after resuture. There was no evidence of metastasis at post mortem.

The fact that a growth of the stomach is $\frac{8}{8}$ already of fair size and extent by the time gastric symptoms bring the patient to his medical attendant raises the question as to whether it is worth while performing radiological investigation of normal people, as in pulmonary tuberculosis. St. John, Swanson and Harvey (1944) found only three tumours in 2,400 normal people over the age of 50 , who were given barium meals. This is clearly not a practical proposition. The close association between pernicious anaemia and carcinoma of the stomach is becoming established bo such findings as those of Rigler, Kaplan and Fink (1945). They investigated radiologically 2 I I cases of pernicious anaemia and found carcinoma in 8 per cent. and polypi in 7.1 per cent. of these. Further, in a review of 43,000 post mortems, there were 293 cases of pernicious anaemia over the age of 45 and, of these, $3^{6}$ had carcinomas-a percentage of 12.3, which is three times the figure for carcinoma of the stomach for the rest of this autopsy series of the same age group. Kaplan and Rigler (1945).

Patients with pernicious anaemia thus form one small group in which there is some hope of discovering carcinoma at an early stage. Experience shows that carcinoma of the stomach may be present when the patient first presents himself with a macrocytic anaemia, during the first two years of treatment or, less commonly, at some later date. Routine roentgenological examination six monthly, at least for the first two years, in cases of pernicious anaemia may well prove worthwhile, and certainly if the anaemia dnes not respond completely to treatment. 
The relation of gastric ulcer to carcinoma is a very vexed one. It is generally agreed that the lowest figure for carcinomata developing in a pre-existing simple ulcer lies between 5 and io per cent. Not the least of the difficulties is the correct microscopical diagnosis of malignancy in a chronic gastric ulcer. At the Mayo Clinic it was routine practice to submit all large gastric ulcers to operation without further delay. In this way some of the better operative survival rates are produced. Gray, Balfour and Kirklin (1934) report a series of I,000 patients at the Mayo Clinic in whom gastric carcinomata were removed, the glands not being involved ; 50 per cent. were alive and well three years later. Where the glands were involved, only 20 . per cent. were alive and well three years later. Taking into account the relapse rate on medical treatment of large gastric ulcers and the small operative mortality for partial gastrectomy in recent years, and the definite risk of carcinoma existing, or soon to follow, in these cases, there seems little doubt that all such large ulcers should be submitted to the surgeon without delay.

In discussing the available means of confirming a suspected diagnosis of carcinoma of the stomach it must be stressed that in practice this rests very largely on examination by a competent radiologist. The other aids to diagnosis are as a rule less reliable and some no more than suggestive and liable to serious fallacies.

The fractional test meal is most fallacious and can almost be dispensed with. While statistically there is achlorhydria or hypochlorhydria in 50 to 70 per cent. of cases there may be a normal or a high acid curve even when the carcinoma is well advanced. In the late age groups as many as 40 per cent. of normal people have hypochlorhydria or achlorhydria so that in the individual case before the clinician the presence or absence of acid has no diagnostic value at all. The assessment of delay in emptying of the stomach by the persistence of starch, etc., is much more reliably revealed by the retention of barium in the stomach on radiological examination. The presence of lactic acid, sarcinae and yeasts merely indicates pyloric obstruction which may be either simple or malignant. Blood may be sucked from the normal mucosa, though the finding of altered blood in all fractions does suggest that the ulceration is malignant rather than simple.

Tests for occult blood are of some value if full allowance is made for possible errors in bleeding from the gums, etc. In carcinoma in the ulcerative stage the benzidine test gives persistently positive results but it may be negative in early cases before ulceration occurs and in certain scirrhus growths. Negative tests do not exclude carcinoma, positive results are commonly due to other causes, but constantly positive findings do strongly suggest an alimentary growth to sum up the very limited value of tests for occult blood.

The blood sedimentation rate of a simple peptic ulcer is normal, or it may be slightly raised if there is associated gastritis. In carcinoma the sedimentation rate is raised roughly according to the size and extent of the growth or according to its degree of malignancy. An early or small growth, however, may give figures within the accepted range of normal. The finding of a sedimentation rate of over $20 \mathrm{~mm}$. in the first hour (Westergren $200 \mathrm{~mm}$. tube) in a case of gastric ulcer shown radiologically is strong presumptive evidence of malignancy:

The gastroscope plays a more important part in diagnosis today, although it has its obvious and serious limitations in the restricted field of vision and in the correct interpretation of the findings. Its value in combination with radiographic examination must be stressed. A gastric ulcer revealed radiologically will sometimes show an edge, or nodules nearby, suggestive of neoplasm, and slight alterations from the normal mucosal pattern at the radiological examination may be confirmed by gastroscopy and lead to the discovery of an early and operable carcinoma. Occasionally a case under suspicion will give a normal barium meal result but the gastroscope will reveal the lesion. The most helpful means of diagnosing carcinoma of the stomach in an operable stage is the prompt and frequent use of the gastroscope combined with radiological examination.

Sometimes the clinical suspicion of carcinoma of the stomach remains so strong that it is necessary to submit the patient to laparotomy even after all these aids to diagnosis have given negative results. 
The prognosis of carcinoma of the stomach is almost uniformly bad. The vast majority of untreated cases die within a year of diagnosis, the remainder die within two years with the exception of the very rare patient who survives even up to five years. Applying the criterion of cure of survival for five or more years after operation few surgeons can claim many cures. On the other hand some lives are prolonged for one to three years in comfort and in useful employment by well judged surgery.

The symptomatic or medical treatment of a patient with this disease does not require to be very strict. The diet is on the lines of a peptic ulcer without undue insistence on the regularity or strictness of that diet. In fact the patient will soon find out for himself that a soft, light diet will suit him best. The more solid foods can be allowed if they do not upset the patient. Occasionally mild dyspepsia is relieved by difute hydrochloric acid in drachm doses with water taken at meals, but, in the majority of cases alkatine powders give more relief, temporary and partial though it may be. Aspirin, or better, a combination of aspirin, phenacetin and caffeine is of great benefit and its use will put off the day for commencing morphine. In the next stage morphine or opium by mouth is added to the aspirin; finally injections of morphine will have to be given and the dose is as much as is necessary to keep the patient comfortable.
Deep X-ray therapy never cures this disease, it very seldom produces any relief of symptoms and often makes the patient much more miserable. In my opinion it plays no part in the treatment of carcinoma of the stomach.

Where there is pyloric obstruction causing troublesome vomiting and inanition, even in a case considered inoperable from the curative point of view, it may be necessary to advise a palliative gastroenterostomy after taking into account the high mortality of such a procedure.

When there is anaemia the appropriate haematinic, liver or iron, may give much symptomatic relief but transfusions of blood should not be given unless there is hope of an operative procedure. The only justification for blood transfusion in inoperable cases is to satisfy a complaining patient or, more often, the relatives.

\section{Summary}

The diagnosis of carcinoma of the stomach is discussed in two parts. When is it to be suspected and, if suspected, how is it to be confirmed ?

The relative values of the different aids to diagnosis are mentioned and the importance of a combination of roentgenological examination with gastroscopy is stressed.

Prognosis and symptomatic treatment are briefly reviewed.

\section{BIBLIOGRAPHY}

GRAY, H. K., BALFOUR, D. C., and KIRKLIN, B. R. (1934) Amer. $\mathcal{F}$. Cancer, $22,249$.

KAPLAN, H. S., and RIGLER, L. S. (1945), Amer. F. Med. Sci., 209, 339.

PLUMMER, N. S. (1931), ' Guy's Hosp. Rep.,' Oct.; 407.

RIGLER, L. S., KAPLAN, H. S.; and FINK, D. L. (1945), F.A.M.A., 128, 426 .

ST. JOHN, F. B., SWANSON, P. C., and HARVEY, H. C. (1944), 